\title{
Equivalent Conditions of Complete Convergence for Weighted Sums of Sequences of Negatively Dependent Random Variables
}

\author{
Mingle Guo \\ School of Mathematics and Computer Science, Anhui Normal University, Wuhu 241003, China \\ Correspondence should be addressed to Mingle Guo, mleguo@163.com
}

Received 29 August 2012; Accepted 14 October 2012

Academic Editor: Josef Diblík

Copyright (c) 2012 Mingle Guo. This is an open access article distributed under the Creative Commons Attribution License, which permits unrestricted use, distribution, and reproduction in any medium, provided the original work is properly cited.

The complete convergence for weighted sums of sequences of negatively dependent random variables is investigated. By applying moment inequality and truncation methods, the equivalent conditions of complete convergence for weighted sums of sequences of negatively dependent random variables are established. These results not only extend the corresponding results obtained by Li et al. (1995), Gut (1993), and Liang (2000) to sequences of negatively dependent random variables, but also improve them.

\section{Introduction}

In many stochastic model, the assumption that random variables are independent is not plausible. Increases in some random variables are often related to decreases in other random variables, so an assumption of negatively dependence is more appropriate than an assumption of independence.

Lehmann [1] introduced the notion of negatively quadrant dependent (NQD) random variables in the bivariate case.

Definition 1.1. Random variables $X$ and $Y$ are said to be NQD if

$$
P(X \leq x, Y \leq y) \leq P(X \leq x) P(Y \leq y)
$$

for all $x, y \in \mathbb{R}$. A sequence of random variables $\left\{X_{n}, n \geq 1\right\}$ is said to be pairwise NQD if for all $i, j(i \neq j), X_{i}$ and $X_{j}$ are NQD. 
It is important to note that (1.1) is equivalent to

$$
P(X>x, Y>y) \leq P(X>x) P(Y>y)
$$

for all $x, y \in \mathbb{R}$. However, (1.1) and (1.2) are not equivalent for a collection of three or more random variables. Consequently, the definition of NQD was extended to the multivariate case by Ebrahimi and Ghosh [2].

Definition 1.2. A finite family of random variables $\left\{X_{i}, 1 \leq i \leq n\right\}$ is said to be negatively dependent (ND) if for all real numbers $x_{1}, x_{2}, \ldots, x_{n}$,

$$
\begin{aligned}
& P\left(\bigcap_{i=1}^{n}\left(X_{i} \leq x_{i}\right)\right) \leq \prod_{i=1}^{n} P\left(X_{i} \leq x_{i}\right), \\
& P\left(\bigcap_{i=1}^{n}\left(X_{i}>x_{i}\right)\right) \leq \prod_{i=1}^{n} P\left(X_{i}>x_{i}\right) .
\end{aligned}
$$

An infinite family of random variables is ND if every finite subfamily is ND.

Negative dependence has been very useful in reliability theory and applications. Since the paper of Ebrahimi and Ghosh [2] appeared, Taylor et al. [3, 4] studied the laws of large numbers for arrays of rowwise ND random variables, Ko and Kim [5] and Ko et al. [6] investigated the strong laws of large numbers for weighted sums of ND random variables. Volodin [7] obtained the Kolmogorov exponential inequality for ND random variables, Amini and Bozorgnia [8] studied the complete convergence for ND random variable sequences, Volodin et al. [9] obtained the convergence rates in the form of a Baum-Katz, and Wu [10] investigated complete convergence for weighted sums of sequences of negatively dependent random variables.

The concept of negatively associated random variables was introduced by Alam and Saxena [11] and carefully studied by Joag-Dev and Proschan [12].

Definition 1.3. A finite family of random variables $\left\{X_{i}, 1 \leq i \leq n\right\}$ is said to be negatively associated (NA), if for every pair disjoint subset $A$ and $B$ of $\{1,2, \ldots, n\}$ and any real nondecreasing coordinate-wise functions $f_{1}$ on $\mathbb{R}^{A}$ and $f_{2}$ on $\mathbb{R}^{B}$,

$$
\operatorname{Cov}\left(f_{1}\left(X_{i}, i \in A\right), f_{2}\left(X_{i}, i \in B\right)\right) \leq 0
$$

whenever the covariance exists. An infinite family of random variables $\left\{X_{i},-\infty<i<\infty\right\}$ is NA if every finite subfamily is NA.

As pointed out and proved by Joag-Dev and Proschan [12], a number of wellknown multivariate distributions posses the NA property, such as multinomial, convolution of unlike multinomial, multivariate hypergeometric, Dirichlet, permutation distribution, random sampling without replacement, and joint distribution of ranks.

Obviously, NA implies ND from the definition of NA and ND. But ND does not imply NA, so ND is much weaker than NA. Hence, the extending the limit properties of 
independent random variables to the case of ND random variables is highly desirable and considerably significant in the theory and application.

The concept of complete convergence of a sequence of random variables was introduced by Hsu and Robbins [13] as follows. A sequence $\left\{X_{n}, n \geq 1\right\}$ of random variables is said to converge completely to a constant $C$ if

$$
\sum_{n=1}^{\infty} P\left(\left|X_{n}-C\right|>\epsilon\right)<\infty \quad \forall \epsilon>0
$$

In view of the Borel-Cantelli lemma, the complete convergence implies almost sure convergence. Therefore, the complete convergence is very important tool in establishing almost sure convergence. When $\left\{X_{n}, n \geq 1\right\}$ is independent and identically distributed (i.i.d), Baum and Katz [14] proved the following remarkable result concerning the convergence rate of the tail probabilities $P\left(\left|S_{n}\right|>\epsilon n^{1 / p}\right)$ for any $\epsilon>0$.

Theorem A. Let $0<p<2$ and $r \geq p$. Then,

$$
\sum_{n=1}^{\infty} n^{r / p-2} P\left(\left|S_{n}\right|>\epsilon n^{1 / p}\right)<\infty \quad \forall \epsilon>0,
$$

if and only if $E\left|X_{1}\right|^{r}<\infty$, where $E X_{1}=0$ whenever $1 \leq p<2$.

There is an interesting and substantial literature of investigation of extending the Baum-Katz Theorem along a variety of different paths. Since partial sums are a particular case of weighted sums and the weighted sums are often encountered in some actual questions, the complete convergence for the weighted sums seems more important. Li et al. [15] discussed the complete convergence for independent weighted sums. Gut [16] discussed complete convergence of Cesàro means of i.i.d random variables. Liang [17] extended the conclusions of Li et al. [15] and Gut [16] to NA random variables and obtained the following results.

Theorem B. Let $\left\{X, X_{n}, n \geq 1\right\}$ be a sequence of identically distributed NA random variables and let $r>1$. Assume $\beta>-1$ and $\left\{a_{n i} \approx(i / n)^{\beta}(1 / n), 1 \leq i \leq n, n \geq 1\right\}$ is a triangular array of real numbers such that $\sum_{i=1}^{n} a_{n i}=1$ for all $n \geq 1$. Then, the following are equivalent:

(i)

$$
\begin{gathered}
E|X|^{(r-1) /(1+\beta)}<\infty, \quad \text { for }-1<\beta<-\frac{1}{r} \\
E|X|^{r} \log (1+|X|)<\infty, \quad \text { for } \beta=-\frac{1}{r} \\
E|X|^{r}<\infty, \quad \text { for } \beta>-\frac{1}{r} \\
E X=0,
\end{gathered}
$$

(ii)

$$
\sum_{n=1}^{\infty} n^{r-2} P\left(\max _{1 \leq k \leq n}\left|\sum_{i=1}^{k} a_{n i} X_{i}\right|>\epsilon\right)<\infty \quad \forall \epsilon>0
$$


Theorem C. Let $\left\{X, X_{n}, n \geq 0\right\}$ be a sequence of identically distributed NA random variables and let $r>1,0<\alpha \leq 1$. Then, the following are equivalent:

(i)

$$
\begin{gathered}
E|X|^{(r-1) / \alpha}<\infty, \quad \text { for } 0<\alpha<1-\frac{1}{r}, \\
E|X|^{r} \log (1+|X|)<\infty, \quad \text { for } \alpha=1-\frac{1}{r} \\
E|X|^{r}<\infty, \quad \text { for } 1-\frac{1}{r}<\alpha \leq 1, \\
E X=0,
\end{gathered}
$$

(ii)

$$
\sum_{n=1}^{\infty} n^{r-2} P\left(\max _{0 \leq k \leq n}\left|\sum_{i=0}^{k} A_{n-i}^{\alpha-1} X_{i}\right|>\epsilon A_{n}^{\alpha}\right)<\infty \quad \forall \epsilon>0
$$

where $A_{n}^{\alpha}=(\alpha+1)(\alpha+2) \cdots(\alpha+n) / n !, n=1,2, \ldots$, and $A_{0}^{\alpha}=1$.

In the current work, we study the complete convergence for ND random variables. Equivalent conditions of complete convergence for weighted sums of sequences of ND random variables are established. As a result, we not only promote and improve the results of Liang [17] for NA random variables to ND random variables without necessarily imposing any extra conditions, but also relax the range of $\beta$.

For the proofs of the main results, we need to restate a few lemmas for easy reference. Throughout this paper, The symbol $C$ denotes a positive constant which is not necessarily the same one in each appearance and $I(A)$ denotes the indicator function of $A$. Let $a_{n} \ll b_{n}$ denote that there exists a constant $C>0$ such that $a_{n} \leq C b_{n}$ for sufficiently large $n$, and let $a_{n} \approx b_{n}$ mean $a_{n} \ll b_{n}$ and $b_{n} \ll a_{n}$. Also, let $\log x$ denote $\ln \max (e, x)$.

Lemma 1.4 (see [11]). Let $\left\{X_{n}, n \geq 1\right\}$ be a sequence of $\mathrm{ND}$ random variables and let $\left\{f_{n}, n \geq 1\right\}$ be a sequence of Borel functions all of which are monotone increasing (or all are monotone decreasing). Then, $\left\{f_{n}\left(X_{n}\right), n \geq 1\right\}$ is still a sequence of ND random variables.

Lemma 1.5 (see [18]). Let $\left\{X_{i}, 1 \leq i \leq n\right\}$ be a sequence of $\mathrm{ND}$ random variables, $E X_{i}=$ $0, E\left|X_{i}\right|^{M}<\infty, 1 \leq i \leq n, M \geq 2$. Then,

$$
E\left|\sum_{i=1}^{n} X_{i}\right|^{M} \leq C\left[\sum_{i=1}^{n} E\left|X_{i}\right|^{M}+\left(\sum_{i=1}^{n} E\left|X_{i}\right|^{2}\right)^{M / 2}\right]
$$

where $C$ depends only on $M$.

Lemma 1.6 (see [10]). Let $\left\{X_{n}, n \geq 1\right\}$ be a sequence of $\mathrm{ND}$ random variables. Then, there exists a positive constant $C$ such that for any $x \geq 0$ and all $n \geq 1$,

$$
\left(1-P\left(\max _{1 \leq i \leq n}\left|X_{i}\right|>x\right)\right)^{2} \sum_{i=1}^{n} P\left(\left|X_{i}\right|>x\right) \leq C P\left(\max _{1 \leq i \leq n}\left|X_{i}\right|>x\right) .
$$


By Lemma 1.2 and Theorem 3 in [19], we can obtain the following lemma.

Lemma 1.7. Let $\left\{X_{i}, 1 \leq i \leq n\right\}$ be a sequence of $\mathrm{ND}$ random variables, $E X_{i}=0, E\left|X_{i}\right|^{M}<\infty, 1 \leq$ $i \leq n, M \geq 2$. Then,

$$
E \max _{1 \leq j \leq n}\left|\sum_{i=1}^{j} X_{i}\right|^{M} \leq C \log ^{M} n\left(\sum_{i=1}^{n} E\left|X_{i}\right|^{M}+\left(\sum_{i=1}^{n} E\left|X_{i}\right|^{2}\right)^{M / 2}\right),
$$

where $C$ depends only on $M$.

By using Fubini's theorem, the following lemma can be easily proved. Here, we omit the details of the proof.

Lemma 1.8. Let $X$ be a random variable, then

(i) $\int_{1}^{\infty} u^{\beta} E|X|^{\alpha} I\left(|X|>u^{\gamma}\right) d u \ll E|X|^{(\beta+1) / \gamma+\alpha}$ for any $\alpha \geq 0, \gamma>0$ and $\beta>-1$;

(ii) $\int_{1}^{\infty} u^{\beta} \log u E|X|^{\alpha} I\left(|X|>u^{\gamma}\right) d u \ll E|X|^{(\beta+1) / \gamma+\alpha} \log (1+|X|)$ for any $\alpha \geq 0, \gamma>0$ and $\beta>-1$.

\section{Main Results}

Now we state our main results. The proofs will be given in Section 3.

Theorem 2.1. Let $\left\{X, X_{n}, n \geq 1\right\}$ be a sequence of identically distributed ND random variables, $r>1, p>1 / 2, \beta+p>0$ and suppose that $E X=0$ for $1 / 2<p \leq 1$. Assume that $\left\{a_{n i} \approx\right.$ $\left.(i / n)^{\beta}\left(1 / n^{p}\right), 1 \leq i \leq n, n \geq 1\right\}$ is a triangular array of real numbers. Then, the following are equivalent:

(i)

$$
\begin{gathered}
E|X|^{(r-1) /(p+\beta)}<\infty, \quad \text { for }-p<\beta<-\frac{p}{r} \\
E|X|^{r / p} \log (1+|X|)<\infty, \quad \text { for } \beta=-\frac{p}{r} \\
E|X|^{r / p}<\infty, \quad \text { for } \beta>-\frac{p}{r}
\end{gathered}
$$

(ii)

$$
\sum_{n=1}^{\infty} n^{r-2} P\left(\max _{1 \leq k \leq n}\left|\sum_{i=1}^{k} a_{n i} X_{i}\right|>\epsilon\right)<\infty \quad \forall \epsilon>0
$$

Theorem 2.2. Let $\left\{X, X_{n}, n \geq 0\right\}$ be a sequence of identically distributed ND random variables, $r>1, p>1 / 2, \beta+p>0$ and suppose that EX $=0$ for $1 / 2<p \leq 1$. Assume that $\left\{a_{n i} \approx\right.$ $\left.((n-i) / n)^{\beta}\left(1 / n^{p}\right), 0 \leq i \leq n-1, n \geq 1\right\}$ is a triangular array of real numbers. Then, the following are equivalent: 
(i)

$$
\begin{gathered}
E|X|^{(r-1) /(p+\beta)}<\infty, \text { for }-p<\beta<-\frac{p}{r} \\
E|X|^{r / p} \log (1+|X|)<\infty, \quad \text { for } \beta=-\frac{p}{r} \\
E|X|^{r / p}<\infty, \text { for } \beta>-\frac{p}{r}
\end{gathered}
$$

(ii)

$$
\sum_{n=1}^{\infty} n^{r-2} P\left(\max _{0 \leq k \leq n-1}\left|\sum_{i=0}^{k} a_{n i} X_{i}\right|>\epsilon\right)<\infty \quad \forall \epsilon>0 .
$$

Remark 2.3. Since NA random variables are a special case of ND random variables, taking $p=1$ in Theorem 2.1, we obtain the result of Liang [17]. Thus, we not only promote and improve the results of Liang [17] for NA random variables to ND random variables without necessarily imposing any extra conditions, but also relax the range of $\beta$.

Remark 2.4. Taking $\beta=0, a_{n i}=1 / n^{p}, 1 \leq i \leq n, n \geq 1$ in Theorem 2.1, we improve the result of Baum and Katz [14].

Corollary 2.5. Let $\left\{X, X_{n}, n \geq 0\right\}$ be a sequence of identically distributed ND random variables, $r>$ $1, p>1 / 2,0<\alpha \leq 1$, and $E X=0$. Let $A_{n}^{\alpha}=(\alpha+1)(\alpha+2) \cdots(\alpha+n) / n !, n=1,2, \ldots$ and $A_{0}^{\alpha}=$ 1. Then, the following are equivalent:

(i)

$$
\begin{gathered}
E|X|^{(r-1) /(p \alpha)}<\infty, \text { for } 0<\alpha<1-\frac{1}{r}, \\
E|X|^{r / p} \log (1+|X|)<\infty, \quad \text { for } \alpha=1-\frac{1}{r}, \\
E|X|^{r / p}<\infty, \quad \text { for } 1-\frac{1}{r}<\alpha \leq 1,
\end{gathered}
$$

(ii)

$$
\sum_{n=1}^{\infty} n^{r-2} P\left(\max _{0 \leq k \leq n}\left|\sum_{i=0}^{k}\left(A_{n-i}^{\alpha-1}\right)^{p} X_{i}\right|>\epsilon\left(A_{n}^{\alpha}\right)^{p}\right)<\infty \quad \forall \epsilon>0
$$

\section{Proofs of the Main Results}

Proof of Theorem 2.1. First, we prove $(2.1) \Rightarrow(2.2)$. Note that $a_{n i}=a_{n i}^{+}-a_{n i}^{-}$, where $a_{n i}^{+}=$ $\max \left(a_{n i}, 0\right)$ and $a_{n i}^{-}=\max \left(-a_{n i}, 0\right)$. Thus, to prove (2.2), it suffices to show that

$$
\sum_{n=1}^{\infty} n^{r-2} P\left(\max _{1 \leq k \leq n}\left|\sum_{i=1}^{k} a_{n i}^{+} X_{i}\right|>\epsilon\right)<\infty, \quad \sum_{n=1}^{\infty} n^{r-2} P\left(\max _{1 \leq k \leq n}\left|\sum_{i=1}^{k} a_{n i}^{-} X_{i}\right|>\epsilon\right)<\infty .
$$


So, without loss of generality, we can assume that $a_{n i}>0,1 \leq i \leq n, n \geq 1$. Choose $\delta>0$ being small enough and sufficient large integer $K$. Let, for every $1 \leq i \leq n, n \geq 1$,

$$
\begin{gathered}
X_{n i}^{(1)}=-n^{-\delta} I\left(a_{n i} X_{i}<-n^{-\delta}\right)+a_{n i} X_{i} I\left(\left|a_{n i} X_{i}\right| \leq n^{-\delta}\right)+n^{-\delta} I\left(a_{n i} X_{i}>n^{-\delta}\right), \\
X_{n i}^{(2)}=\left(a_{n i} X_{i}-n^{-\delta}\right) I\left(n^{-\delta}<a_{n i} X_{i}<\frac{\epsilon}{K}\right) \\
X_{n i}^{(3)}=\left(a_{n i} X_{i}+n^{-\delta}\right) I\left(-\frac{\epsilon}{K}<a_{n i} X_{i}<-n^{-\delta}\right) \\
X_{n i}^{(4)}=\left(a_{n i} X_{i}+n^{-\delta}\right) I\left(a_{n i} X_{i} \leq-\frac{\epsilon}{K}\right)+\left(a_{n i} X_{i}-n^{-\delta}\right) I\left(a_{n i} X_{i} \geq \frac{\epsilon}{K}\right) .
\end{gathered}
$$

Obviously, $\sum_{i=1}^{k} a_{n i} X_{i}=\sum_{i=1}^{k} X_{n i}^{(1)}+\sum_{i=1}^{k} X_{n i}^{(2)}+\sum_{i=1}^{k} X_{n i}^{(3)}+\sum_{i=1}^{k} X_{n i}^{(4)}$. Note that

$$
\left(\max _{1 \leq k \leq n}\left|\sum_{i=1}^{k} a_{n i} X_{i}\right|>4 \epsilon\right) \subset \bigcup_{j=1}^{4}\left(\max _{1 \leq k \leq n}\left|\sum_{i=1}^{k} X_{n i}^{(j)}\right|>\epsilon\right) .
$$

Thus, in order to prove (2.2), it suffices to show that

$$
I_{j}=: \sum_{n=1}^{\infty} n^{r-2} P\left(\max _{1 \leq k \leq n}\left|\sum_{i=1}^{k} X_{n i}^{(j)}\right|>\epsilon\right)<\infty, \quad j=1,2,3,4
$$

By the definition of $X_{n i}^{(4)}$, we see that $\left(\max _{1 \leq k \leq n}\left|\sum_{i=1}^{k} X_{n i}^{(4)}\right|>\epsilon\right) \subset\left(\max _{1 \leq i \leq n}\left|a_{n i} X_{i}\right|>\epsilon / K\right)$. Since $a_{n i} \approx(i / n)^{\beta}\left(1 / n^{p}\right)$, by Lemma 1.8 , we have

$$
\begin{aligned}
I_{4} & \leq \sum_{n=1}^{\infty} n^{r-2} \sum_{i=1}^{n} P\left(\left|a_{n i} X_{i}\right|>\frac{\epsilon}{K}\right) \leq \sum_{n=1}^{\infty} n^{r-2} \sum_{i=1}^{n} P\left(|X|>\frac{\epsilon}{C K} n^{p+\beta} i^{-\beta}\right) \\
& \approx \int_{1}^{\infty} x^{r-2} \int_{1}^{x} P\left(|X|>\frac{\epsilon}{C K} x^{p+\beta} y^{-\beta}\right) d y d x \quad\left(\text { letting } u=x^{p+\beta} y^{-\beta}, v=y\right) \\
& =\frac{1}{p+\beta} \int_{1}^{\infty} d u \int_{1}^{u^{1 / p}} u^{(r-1) /(p+\beta)-1} v^{\beta(r-1) /(p+\beta)} P\left(|X|>\frac{\epsilon}{C K} u\right) d v \\
& \approx\left\{\begin{array}{l}
\int_{1}^{\infty} u^{(r-1) /(p+\beta)-1} P\left(|X|>\frac{\epsilon}{C K} u\right) d u \ll E|X|^{(r-1) /(p+\beta)} \quad \text { for }-p<\beta<-\frac{p}{r}, \\
\int_{1}^{\infty} u^{r / p-1} \log u P\left(|X|>\frac{\epsilon}{C K} u\right) d u \ll E|X|^{r / p} \log (1+|X|) \quad \text { for } \beta=-\frac{p}{r}, \\
\int_{1}^{\infty} u^{r / p-1} P\left(|X|>\frac{\epsilon}{C K} u\right) d u \ll E|X|^{r / p} \quad \text { for } \beta>-\frac{p}{r} .
\end{array}\right.
\end{aligned}
$$


Therefore, by (2.1), $I_{4}<\infty$. From the definition of $X_{n i}^{(2)}$, we know $X_{n i}^{(2)}>0$. By using the definition of ND family, we have

$$
\begin{aligned}
& P\left(\max _{1 \leq k \leq n}\left|\sum_{i=1}^{k} X_{n i}^{(2)}\right|>\epsilon\right) \\
& \quad=P\left(\sum_{i=1}^{n} X_{n i}^{(2)}>\epsilon\right) \\
& \quad \leq P\left(\text { there are at least } K \text { indices } i \in[1, n] \text { such that } a_{n i} X_{i}>n^{-\delta}\right) \\
& \quad \leq \sum_{1 \leq i_{1}<i_{2}<\cdots<i_{K} \leq n} \prod_{j=1}^{K} P\left(a_{n i_{j}} X_{i_{j}}>n^{-\delta}\right) \leq\left(\sum_{j=1}^{n} P\left(a_{n j} X>n^{-\delta}\right)\right)^{K} .
\end{aligned}
$$

Since (2.1) implies $E|X|^{r / p}<\infty$, by Markov's inequality and (3.6), we obtain

$$
\begin{aligned}
I_{2} & \leq \sum_{n=1}^{\infty} n^{r-2}\left(\sum_{j=1}^{n} P\left(a_{n j} X>n^{-\delta}\right)\right)^{K} \\
& \leq \sum_{n=1}^{\infty} n^{r-2}\left(\sum_{j=1}^{n} n^{r \delta / p}\left|a_{n j}\right|^{r / p} E|X|^{r / p}\right)^{K} \\
& \approx\left\{\begin{array}{l}
\sum_{n=1}^{\infty} n^{r-2-K r(p+\beta-\delta) / p}, \text { for }-p<\beta<-\frac{p}{r}, \\
\sum_{n=1}^{\infty} n^{r-2-K(r-1-r \delta / p)} \log n, \quad \text { for } \beta=-\frac{p}{r}, \\
\sum_{n=1}^{\infty} n^{r-2-K(r-1-r \delta / p)}, \text { for } \beta>-\frac{p}{r} .
\end{array}\right.
\end{aligned}
$$

Noting that $r>1, p+\beta>0$, we can choose $\delta$ being small enough and sufficient large integer $K$ such that $r-2-K r(p+\beta-\delta) / p<-1$ and $r-2-K(r-1-r \delta / p)<-1$. Thus, by (3.7), we get $I_{2}<\infty$. Similarly, we can obtain $I_{3}<\infty$. In order to estimate $I_{1}$, we first verify that

$$
\max _{1 \leq k \leq n}\left|\sum_{i=1}^{k} E X_{n i}^{(1)}\right| \longrightarrow 0 \quad \text { as } n \longrightarrow \infty .
$$

Note that (2.1) implies $E|X|^{r / p}<\infty$ and $E|X|^{1 / p}<\infty$. When $p>1$, noting that $\left|X_{n i}^{(1)}\right| \leq n^{-\delta}$ and $\left|X_{n i}^{(1)}\right| \leq\left|a_{n i} X_{i}\right|$, by Hölder's inequality we have

$$
\begin{aligned}
\max _{1 \leq k \leq n}\left|\sum_{i=1}^{k} E X_{n i}^{(1)}\right| & \leq \sum_{i=1}^{n} E\left|X_{n i}^{(1)}\right| \\
& \leq n^{-\delta(1-(1 / p))} \sum_{i=1}^{n} E\left|a_{n i} X_{i}\right|^{1 / p} \ll n^{-\delta(1-1 / p)} \sum_{i=1}^{n}\left|a_{n i}\right|^{1 / p}
\end{aligned}
$$


Abstract and Applied Analysis

$$
\begin{aligned}
& \leq n^{-\delta(1-1 / p)} n^{(r-1) / r}\left(\sum_{i=1}^{n}\left|a_{n i}\right|^{r / p}\right)^{1 / r} \ll n^{-\delta(1-1 / p)+1-1 / r}\left(\sum_{i=1}^{n} n^{-r(p+\beta) / p_{i} \beta r / p}\right)^{1 / r} \\
& \approx\left\{\begin{array}{l}
n^{-\delta(1-1 / p)-1 / r-\beta / p}, \quad \text { for }-p<\beta<-\frac{p}{r} \\
n^{-\delta(1-1 / p)} \log n, \quad \text { for } \beta=-\frac{p}{r} \\
n^{-\delta(1-1 / p)}, \quad \text { for } \beta>-\frac{p}{r}
\end{array}\right. \\
& \longrightarrow 0 \quad \text { as } n \longrightarrow \infty .
\end{aligned}
$$

When $1 / 2<p \leq 1$, noting that $E X=0$, by choosing $\delta$ being small enough such that $-\delta(1-$ $r / p)+1-r<0$, we have

$$
\begin{aligned}
\max _{1 \leq k \leq n}\left|\sum_{i=1}^{k} E X_{n i}^{(1)}\right| & \leq 2 \sum_{i=1}^{n} E\left|a_{n i} X_{i}\right| I\left(\left|a_{n i} X_{i}\right|>n^{-\delta}\right) \leq 2 n^{-\delta(1-r / p)} \sum_{i=1}^{n} E\left|a_{n i} X_{i}\right|^{r / p} \\
& \ll n^{-\delta(1-r / p)} \sum_{i=1}^{n}\left|a_{n i}\right|^{r / p} \ll n^{-\delta(1-r / p)}\left(\sum_{i=1}^{n} n^{-r(p+\beta) / p} \beta^{\beta r / p}\right) \\
& \approx\left\{\begin{array}{l}
n^{-\delta-r(p+\beta-\delta) / p}, \text { for }-p<\beta<-\frac{p}{r}, \\
n^{-\delta(1-r / p)+1-r} \log n, \quad \text { for } \beta=-\frac{p}{r}, \\
n^{-\delta(1-r / p)+1-r}, \text { for } \beta>-\frac{p}{r}
\end{array}\right. \\
& \longrightarrow 0 \quad \text { as } n \longrightarrow \infty .
\end{aligned}
$$

Therefore, to prove $I_{1}<\infty$, it suffices to prove that

$$
I_{1}^{*}=: \sum_{n=1}^{\infty} n^{r-2} P\left(\max _{1 \leq k \leq n}\left|\sum_{i=1}^{k}\left(X_{n i}^{(1)}-E X_{n i}^{(1)}\right)\right|>\epsilon\right)<\infty
$$

Note that $\left\{X_{n i}^{(1)}, 1 \leq i \leq n, n \geq 1\right\}$ is still ND by Lemma 1.4. Using Markov's inequality, $C_{r}$ inequality, and Lemma 1.7, we get for a suitably large $M$, which will be determined later,

$$
P\left(\max _{1 \leq k \leq n}\left|\sum_{i=1}^{k}\left(X_{n i}^{(1)}-E X_{n i}^{(1)}\right)\right|>\epsilon\right) \ll(\log n)^{M}\left(\sum_{i=1}^{n} E\left|X_{n i}^{(1)}\right|^{M}+\left(\sum_{i=1}^{n} E\left(X_{n i}^{(1)}\right)^{2}\right)^{M / 2}\right) .
$$


Choosing sufficient large $M$ such that $-2-\delta M+r(\delta-\beta) / p<-1,-1-(M-r / p) \delta<-1$, we have

$$
\begin{aligned}
\sum_{n=1}^{\infty} n^{r-2}(\log n)^{M} \sum_{i=1}^{n} E\left|X_{n i}^{(1)}\right|^{M} & \ll \sum_{n=1}^{\infty} n^{r-2} n^{-\delta(M-r / p)}(\log n)^{M} \sum_{i=1}^{n}\left|a_{n i}\right|^{r / p} \\
& \ll \sum_{n=1}^{\infty} n^{r-2-\delta(M-r / p)}(\log n)^{M} \sum_{i=1}^{n} i^{r \beta / p} n^{-r(p+\beta) / p} \\
& \ll \begin{cases}\sum_{n=1}^{\infty} n^{-2-\delta M+r(\delta-\beta) / p}(\log n)^{M}, \quad \text { for }-p<\beta<-\frac{p}{r}, \\
\sum_{n=1}^{\infty} n^{-1-(M-r / p) \delta}(\log n)^{M+1}, \text { for } \beta=-\frac{p}{r}, \\
\sum_{n=1}^{\infty} n^{-1-(M-r / p) \delta}(\log n)^{M}, \text { for } \beta>-\frac{p}{r}\end{cases} \\
& <\infty .
\end{aligned}
$$

When $r / p \geq 2$, (2.1) implies $E X^{2}<\infty$. Noting that $p+\beta>0, p>1 / 2$, we can choose sufficient large $M$ such that $r-2-M(p+\beta)<-1, r-2-(2 p-1) M / 2<-1$. Then,

$$
\begin{aligned}
\sum_{n=1}^{\infty} n^{r-2}(\log n)^{M}\left(\sum_{i=1}^{n} E\left(X_{n i}^{(1)}\right)^{2}\right)^{M / 2} & \ll \sum_{n=1}^{\infty} n^{r-2}(\log n)^{M}\left(\sum_{i=1}^{n} a_{n i}^{2}\right)^{M / 2} \\
& \ll \sum_{n=1}^{\infty} n^{r-2}(\log n)^{M}\left(\sum_{i=1}^{n} i^{2 \beta} n^{-2(p+\beta)}\right)^{M / 2} \\
& \ll\left\{\begin{array}{l}
\sum_{n=1}^{\infty} n^{r-2-M(p+\beta)}(\log n)^{M}, \text { for }-p<\beta<-\frac{1}{2}, \\
\sum_{n=1}^{\infty} n^{r-2-(2 p-1) M / 2}(\log n)^{3 M / 2}, \quad \text { for } \beta=-\frac{1}{2}, \\
\sum_{n=1}^{\infty} n^{r-2-(2 p-1) M / 2}(\log n)^{M}, \quad \text { for } \beta>-\frac{1}{2}
\end{array}\right. \\
& <\infty .
\end{aligned}
$$

When $r / p<2$, choosing sufficient large $M$ such that $r-2-[\delta(2-r / p)+r(p+\beta) / p] M / 2<$ $-1, r-2-[\delta(2-r / p)+r-1] M / 2<-1$, we have

$$
\begin{aligned}
\sum_{n=1}^{\infty} n^{r-2}(\log n)^{M}\left(\sum_{i=1}^{n} E\left(X_{n i}^{(1)}\right)^{2}\right)^{M / 2} & \\
& \ll \sum_{n=1}^{\infty} n^{r-2} n^{-\delta(2-r / p) M / 2}(\log n)^{M}\left(\sum_{i=1}^{n} a_{n i}^{r / p}\right)^{M / 2}
\end{aligned}
$$


Abstract and Applied Analysis

$$
\begin{aligned}
& \ll \sum_{n=1}^{\infty} n^{r-2} n^{-\delta(2-r / p) M / 2}(\log n)^{M}\left(\sum_{i=1}^{n} i^{r \beta / p} n^{-r(p+\beta) / p}\right)^{M / 2} \\
& \ll\left\{\begin{array}{l}
\sum_{n=1}^{\infty} n^{r-2-[\delta(2-r / p)+r(p+\beta) / p] M / 2}(\log n)^{M}, \quad \text { for }-p<\beta<-\frac{p}{r}, \\
\sum_{n=1}^{\infty} n^{r-2-[\delta(2-r / p)+r-1] M / 2}(\log n)^{3 M / 2}, \text { for } \beta=-\frac{p}{r}, \\
\sum_{n=1}^{\infty} n^{r-2-[\delta(2-r / p)+r-1] M / 2}(\log n)^{M}, \text { for } \beta>-\frac{p}{r}
\end{array}\right. \\
& <\infty .
\end{aligned}
$$

Thus, by (3.13), (3.14), and (3.15), we have $I_{1}^{*}<\infty$.

Now, we proceed to prove $(2.2) \Rightarrow(2.1)$. Since $\max _{1 \leq k \leq n}\left|a_{n k} X_{k}\right| \leq 2 \max _{1 \leq k \leq n}\left|\sum_{i=1}^{k} a_{n i} X_{i}\right|$, by (2.2), we have

$$
\sum_{n=1}^{\infty} n^{r-2} P\left(\max _{1 \leq k \leq n}\left|a_{n k} X_{k}\right|>\epsilon\right)<\infty
$$

Next, we shall prove

$$
P\left(\max _{1 \leq k \leq n}\left|a_{n k} X_{k}\right|>\epsilon\right) \longrightarrow 0 \quad \text { as } n \longrightarrow \infty .
$$

In fact, when $r \geq 2$, (3.16) obviously implies (3.17). When $1<r<2$, noting that $a_{n i} \approx$ $(i / n)^{\beta}\left(1 / n^{p}\right)$, by (3.16), we obtain

$$
\sum_{n=1}^{\infty} n^{r-2} P\left(\max _{1 \leq k \leq n}\left|k^{\beta} X_{k}\right|>\epsilon n^{p+\beta}\right)<\infty \quad \forall \epsilon>0 .
$$

Obviously, $\left\{\max _{1 \leq k \leq n}\left|k^{\beta} X_{k}\right|, n \geq 1\right\}$ is a nondecreasing sequence of nonnegative random variables. By (3.18), we have

$$
\begin{aligned}
\infty & >\sum_{n=1}^{\infty} n^{r-2} P\left(\max _{1 \leq k \leq n}\left|k^{\beta} X_{k}\right|>\epsilon n^{p+\beta}\right)=\sum_{j=0}^{\infty} \sum_{n=2^{j}}^{2^{j+1}-1} n^{r-2} P\left(\max _{1 \leq k \leq n}\left|k^{\beta} X_{k}\right|>\epsilon n^{p+\beta}\right) \\
& \geq \sum_{j=0}^{\infty} 2^{j} 2^{(j+1)(r-2)} P\left(\max _{1 \leq k \leq 2^{j}}\left|k^{\beta} X_{k}\right|>\epsilon 2^{(j+1)(p+\beta)}\right) \\
& =2^{r-2} \sum_{j=0}^{\infty} 2^{j(r-1)} P\left(\max _{1 \leq k \leq 2^{j}}\left|k^{\beta} X_{k}\right|>\epsilon 2^{1+\beta} 2^{j(p+\beta)}\right) .
\end{aligned}
$$

Noting that $r-1>0$, by (3.19), we have

$$
\lim _{j \rightarrow \infty} P\left(\max _{1 \leq k \leq 2^{j}}\left|k^{\beta} X_{k}\right|>\epsilon_{1} 2^{j(p+\beta)}\right)=0 \quad \forall \epsilon_{1}>0 .
$$


Now, for each $n \geq 1$, let $j$ be such that $2^{j} \leq n<2^{j+1}-1$. Then, for all $\epsilon>0$

$$
\begin{aligned}
P\left(\max _{1 \leq k \leq n}\left|k^{\beta} X_{k}\right|>\epsilon n^{p+\beta}\right) & \leq P\left(\max _{1 \leq k \leq 2^{j+1}}\left|k^{\beta} X_{k}\right|>\epsilon 2^{j(p+\beta)}\right) \\
& =P\left(\max _{1 \leq k \leq 2^{j+1}}\left|k^{\beta} X_{k}\right|>\epsilon 2^{-(1+\beta)} 2^{(j+1)(p+\beta)}\right) \longrightarrow 0 \quad \text { as } n \longrightarrow \infty .
\end{aligned}
$$

Noting that $a_{n i} \approx(i / n)^{\beta}\left(1 / n^{p}\right)$, we have

$$
P\left(\max _{1 \leq k \leq n}\left|a_{n k} X_{k}\right|>\epsilon\right) \leq P\left(\max _{1 \leq k \leq n}\left|k^{\beta} X_{k}\right|>\frac{\epsilon}{C} n^{p+\beta}\right) .
$$

Therefore, by (3.21) and (3.22), we get that (3.17) holds.

Thus, by (3.17) and Lemma 1.6, we have

$$
\sum_{i=1}^{n} P\left(\left|a_{n i} X_{i}\right|>\epsilon\right) \ll P\left(\max _{1 \leq k \leq n}\left|a_{n k} X_{k}\right|>\epsilon\right) .
$$

Now (3.16) and (3.23) yield

$$
\sum_{n=1}^{\infty} n^{r-2} \sum_{i=1}^{n} P\left(\left|a_{n i} X_{i}\right|>\epsilon\right)<\infty
$$

By the process of proof of (3.5), we see that (3.24) is equivalent to (2.1).

Proof of Theorem 2.2. The proof is similar to that of Theorem 2.1 and is omitted.

Proof of Corollary 2.5. Put $a_{n i}=\left(A_{n-i}^{\alpha-1} / A_{n}^{\alpha}\right)^{p}, 0 \leq i \leq n, n \geq 1$. Note that, for $\alpha>-1, A_{n}^{\alpha} \approx$ $n^{\alpha} / \Gamma(\alpha+1)$. Therefore, for $\alpha>0$, we obtain $a_{n i} \approx(n-i)^{p(\alpha-1)} n^{-p \alpha}, 0 \leq i<n, n \geq 1, a_{n n} \approx n^{-p \alpha}$. Thus, letting $\beta=p(\alpha-1)$ in Theorem 2.2, we can conclude that (2.5) is equivalent to (2.6).

\section{Acknowledgments}

The research is supported by the National Natural Science Foundation of China (nos. 11271020 and 11201004), the Key Project of Chinese Ministry of Education (nos. 211077) and the Anhui Provincial Natural Science Foundation (nos. 10040606Q30 and 1208085MA11).

\section{References}

[1] E. L. Lehmann, "Some concepts of dependence," Annals of Mathematical Statistics, vol. 37, no. 5, pp. 1137-1153, 1966.

[2] N. Ebrahimi and M. Ghosh, "Multivariate negative dependence," Communications in Statistics, vol. 10, no. 4, pp. 307-337, 1981. 
[3] R. L. Taylor, R. F. Patterson, and A. Bozorgnia, "Weak laws of large numbers for arrays of rowwise negatively dependent random variables," Journal of Applied Mathematics and Stochastic Analysis, vol. 14, no. 3, pp. 227-236, 2001.

[4] R. L. Taylor, R. F. Patterson, and A. Bozorgnia, "A strong law of large numbers for arrays of rowwise negatively dependent random variables," Stochastic Analysis and Applications, vol. 20, no. 3, pp. 643656, 2002.

[5] M.-H. Ko and T.-S. Kim, "Almost sure convergence for weighted sums of negatively orthant dependent random variables," Journal of the Korean Mathematical Society, vol. 42, no. 5, pp. 949-957, 2005.

[6] M.-H. Ko, K.-H. Han, and T.-S. Kim, "Strong laws of large numbers for weighted sums of negatively dependent random variables," Journal of the Korean Mathematical Society, vol. 43, no. 6, pp. 1325-1338, 2006.

[7] A. Volodin, "On the Kolmogorov exponential inequality for negatively dependent random variables," Pakistan Journal of Statistics, vol. 18, no. 2, pp. 249-253, 2002.

[8] D. M. Amini and A. Bozorgnia, "Complete convergence for negatively dependent random variables," Journal of Applied Mathematics and Stochastic Analysis, vol. 16, no. 2, pp. 121-126, 2003.

[9] A. Volodin, M. O. Cabrera, and T. C. Hu, "Convergence rate of the dependent bootstrapped means," Theory of Probability and Its Applications, vol. 50, no. 2, pp. 337-346, 2006.

[10] Q. Wu, "Complete convergence for weighted sums of sequences of negatively dependent random variables," Journal of Probability and Statistics, vol. 2011, Article ID 202015, 16 pages, 2011.

[11] K. Alam and K. M. L. Saxena, "Positive dependence in multivariate distributions," Communications in Statistics, vol. 10, no. 12, pp. 1183-1196, 1981.

[12] K. Joag-Dev and F. Proschan, "Negative association of random variables, with applications," The Annals of Statistics, vol. 11, no. 1, pp. 286-295, 1983.

[13] P. L. Hsu and H. Robbins, "Complete convergence and the law of large numbers," Proceedings of the National Academy of Sciences of the United States of America, vol. 33, pp. 25-31, 1947.

[14] L. E. Baum and M. Katz, "Convergence rates in the law of large numbers," Transactions of the American Mathematical Society, vol. 120, no. 1, pp. 108-123, 1965.

[15] D. L. Li, M. B. Rao, T. F. Jiang, and X. C. Wang, "Complete convergence and almost sure convergence of weighted sums of random variables," Journal of Theoretical Probability, vol. 8, no. 1, pp. 49-76, 1995.

[16] A. Gut, "Complete convergence and Cesàro summation for i.i.d. random variables," Probability Theory and Related Fields, vol. 97, no. 1-2, pp. 169-178, 1993.

[17] H.-Y. Liang, "Complete convergence for weighted sums of negatively associated random variables," Statistics and Probability Letters, vol. 48, no. 4, pp. 317-325, 2000.

[18] N. Asadian, V. Fakoor, and A. Bozorgnia, "Rosenthal's type inequalities for negatively orthant dependent random variables," Journal of the Iranian Statistical Society, vol. 5, no. 1, pp. 66-75, 2006.

[19] F. Móricz, "Moment inequalities and the strong laws of large numbers," Zeitschrift für Wahrscheinlichkeitstheorie und Verwandte Gebiete, vol. 35, no. 4, pp. 299-314, 1976. 


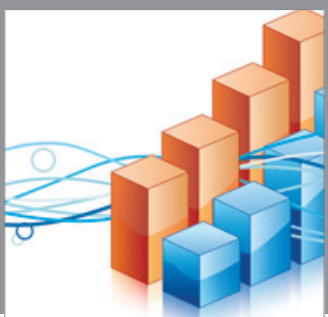

Advances in

Operations Research

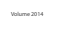

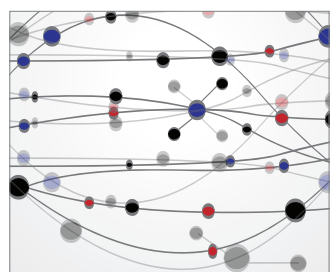

\section{The Scientific} World Journal
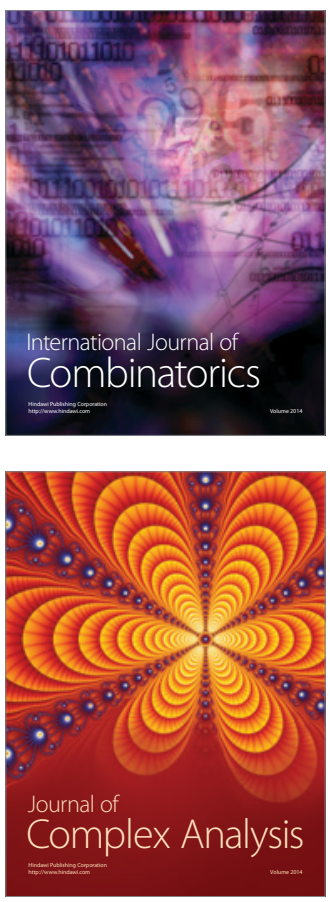

International Journal of

Mathematics and

Mathematical

Sciences
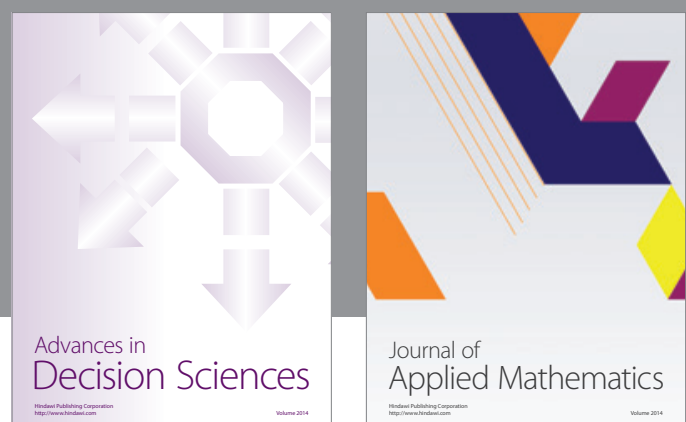

Journal of

Applied Mathematics
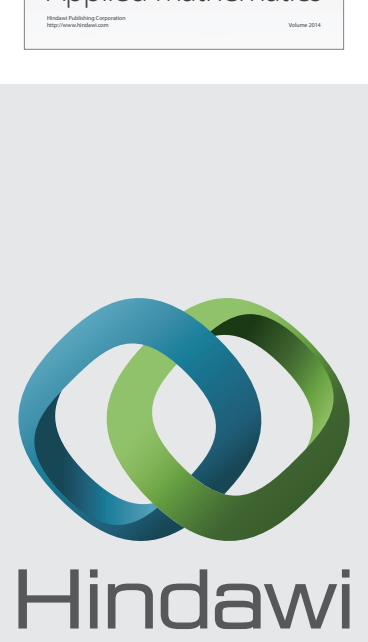

Submit your manuscripts at http://www.hindawi.com
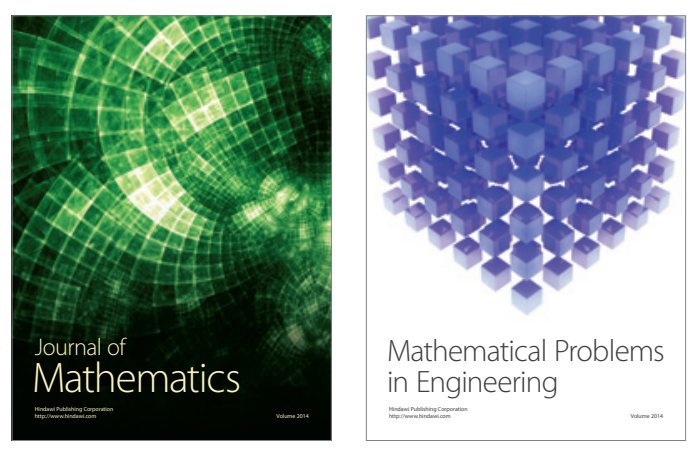

Mathematical Problems in Engineering
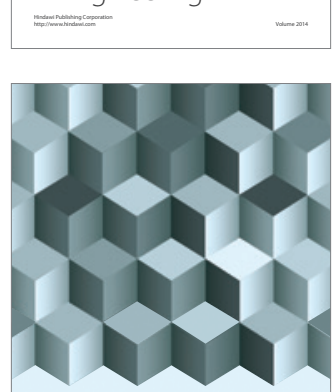

Journal of

Function Spaces
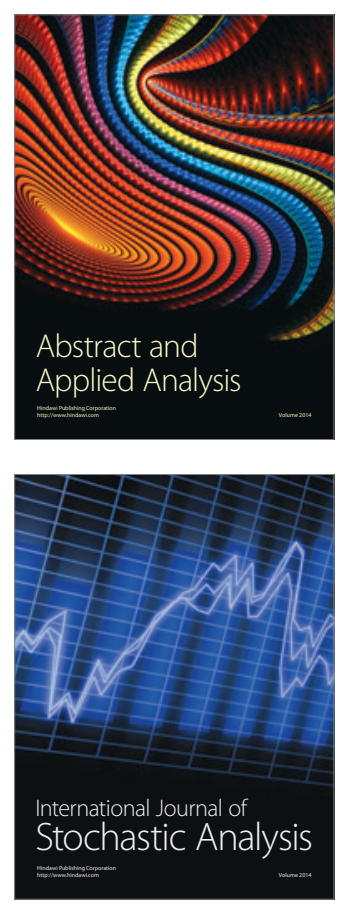

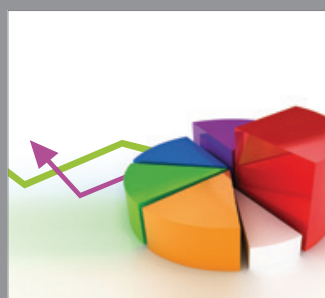

ournal of

Probability and Statistics

Promensencen
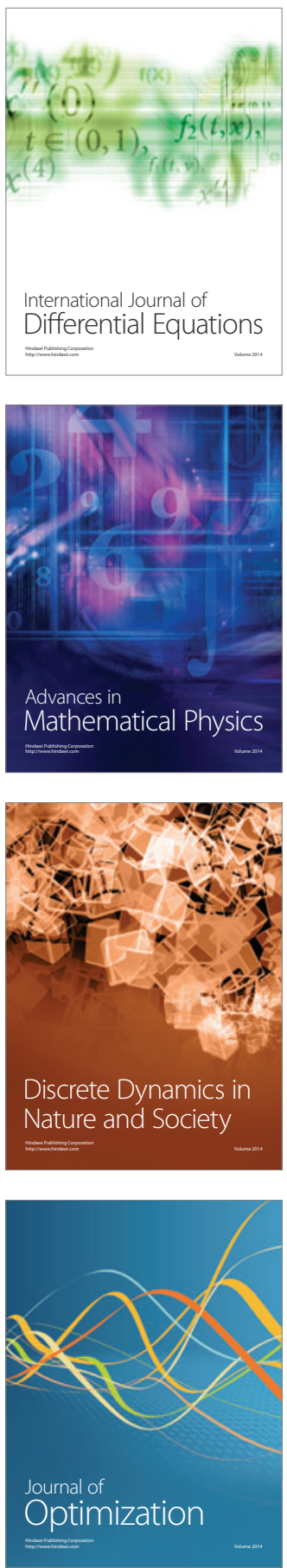